\title{
Priapisme aigu drépanocytaire : analyse de 20 cas traités au CHU de Brazzaville
}

\author{
Acute sickle cell priapism: Analysis of 20 cases treated at the university Hospital of Brazzaville
}

\author{
P.A. Bouya - A.W.S Odzébé $\cdot$ R.D. Koumou Moritoua $\cdot$ A. Elira Dokékias
}

Reçu le 2 avril 2010 ; accepté le 14 octobre 2010

(C) SALF et Springer-Verlag France 2010

Résumé Objectif : Évaluer les résultats des traitements médicaux et chirurgicaux du priapisme drépanocytaire.

Malades et méthodes : Vingt malades ont été traités pour priapisme drépanocytaire dans les services d'urologieandrologie et d'hématologie du CHU de Brazzaville, du $1^{\text {er }}$ janvier 1995 au 31 décembre 2005. Les méthodes thérapeutiques ont été les injections intracaverneuses d'étiléfrine et les fistules cavernospongieuses.

Résultats : 1) Cinq patients traités sans succès par transfusion et étiléfrine orale ont été secondairement traités avec succès par la technique de Winter ; 2) sur cinq patients traités par injection intracaverneuse d'étiléfrine en première intention, on note trois succès et deux échecs, traités avec succès par la technique de Winter ; 3) sur dix patients traités par fistules cavernospongieuses selon Winter en première intention, trois ont récidivé et ont été secondairement traités avec succès par la méthode d'Al-Ghorab. Les résultats fonctionnels à long terme sont : $25 \%$ d'absence d'érection, $25 \%$ d'insuffisance d'érection et $50 \%$ d'érection normale.

Conclusion : Le traitement par injection intracaverneuse d'étiléfrine est efficace avant la $24^{\mathrm{e}}$ heure. Au-delà, les fistules cavernospongieuses donnent les meilleurs résultats. Pour citer cette revue : Andrologie 21 (2011).

Mots clés Priapisme · Drépanocytose · Étiléfrine · Fistule cavernospongieuse

\author{
Abstract Objective: To evaluate the results of medical and \\ surgical treatment of sickle cell priapism. \\ P.A. Bouya · A.W.S Odzébé $(\varangle) \cdot$ R.D. Koumou Moritoua \\ Service d'urologie-andrologie, \\ CHU de Brazzaville, \\ BP 2522 Brazzaville, Congo \\ e-mail : odzebe_s@yahoo.fr \\ A. Elira Dokékias \\ Service d'hématologie, \\ CHU de Brazzaville, BP 32, \\ Brazzaville, Congo
}

Patients and methods: Between 01 January, 1995 and 31 December, 2005, 20 patients were treated for priapism in the services of Urology-Andrology and Haematology of the University Hospital, Brazzaville. Treatment included intracavernous injection of etilefrine and cavernospongious fistula.

Results: 1) Five patients initially treated with transfusion and oral etilefrine without success were successfully cured after using the technique of Winter. 2) In 5 patients treated with intracavernous injection of etilefrine as first-line, 3 were cured and two failed, who were later cured using the technique of Winter. 3) Of 10 patients treated by Winter cavernospongious fistulas as first-line, 3 had recurrences and secondarily were successfully treated by the method of Al-Ghorab. Functional results in the long term include 25\% lack of erection, $25 \%$ of erection failure and $50 \%$ of normal erection.

Conclusion: Treatment with intracavernous injection of etilefrine is effective before the 24th hour. Beyond this time, cavernospongious fistula yield better results. To cite this journal: Andrologie 21 (2011).

Keywords Priapism - Sickle cell anemia Etilefrine Cavernospongious fistulas

\section{Introduction}

Le priapisme, dont le terme Priape dérive de la mythologie antique symbole de la virilité et de la fécondité chez les Romains [1], correspond à une érection prolongée douloureuse n'aboutissant pas à l'éjaculation et évoluant spontanément vers l'impuissance définitive.

Au Congo, les étiologies sont dominées par la drépanocytose [2] dont la forme homozygote a une fréquence estimée à $1,25 \%[3,4]$.

La prise en charge thérapeutique est fonction du mécanisme physiopathologique. Dans la drépanocytose, il s'agit 
souvent d'un priapisme de bas débit, bien que quelques rares cas de haut débit soient rapportés dans la littérature [5]. Les travaux sur l'histoire naturelle de la maladie drépanocytaire et la caractérisation du génome humain nous offrent actuellement l'occasion de comprendre les rapports entre les facteurs environnementaux et les hématies chez le drépanocytaire [6].

Ainsi, le priapisme drépanocytaire peut bénéficier d'un traitement non chirurgical quand le diagnostic est précoce. Notre étude a pour but d'évaluer les résultats des traitements médical et chirurgical du priapisme drépanocytaire.

\section{Patients et méthodes}

Il s'agit d'une étude rétrospective réalisée dans les services d'urologie-andrologie et d'hématologie du CHU de Brazzaville, du $1^{\text {er }}$ janvier 1995 au 31 décembre 2005.

Durant cette période de 11 ans, 32 patients ont été pris en charge pour priapisme aigu drépanocytaire. Pour chaque patient inclus dans l'étude, le dossier comprenait : une observation médicale avec un bilan biologique (hémogramme, électrophorèse de l'hémoglobine), un compte rendu du traitement médical, un compte rendu opératoire, les suites opératoires immédiates et à distance. Vingt patients ont été retenus. Douze patients ayant des dossiers incomplets ont été exclus.

Les méthodes thérapeutiques utilisées ont été les suivantes :

- le traitement médical par voie générale, qui a consisté en une hydratation, une administration des anti-infectieux et la transfusion sanguine (échange transfusionnel partiel ou transfusion simple de concentrés érythrocytaires). Le traitement spécifique réalisait une ponction du corps caverneux avec une aiguille de Butterfly $\mathrm{n}^{\circ} 19 \mathrm{G}$ après injection de xylocaïne $1 \%$. On observait l'écoulement du sang noir, ensuite rouge, suivi de l'injection de l'étiléfrine. Cette méthode thérapeutique était indiquée dans les cas de priapisme évoluant depuis moins de 30 heures ;

- sur le plan chirurgical, deux techniques ont été utilisées :

- une fistule spongiocaverneuse transglandulaire à ciel fermé selon la technique de Winter (à l'aide d'un trocart $16 \mathrm{G}$ réalisant un tunnel entre le gland et chacun des corps caverneux) ;

- une fistule spongiocaverneuse à ciel ouvert par incision glandulaire selon la technique d'Al-Ghorab ;

- le succès était obtenu en cas de détumescence durable alors que la détumescence non obtenue ou non durable (récidive précoce) était considérée comme un échec ;

- la prévention des récidives a été faite par administration orale d'étiléfrine.
L'évaluation de la fonction érectile était faite lors d'une consultation réalisée à 1,6 et 12 mois après le traitement. L'interrogatoire recherchait l'existence des érections matinales ou pendant le sommeil chez les enfants, et chez les adultes, l'existence des érections permettant un rapport sexuel. Les patients étaient ainsi classés en trois groupes :

- groupe 1: une absence d'érection;

- groupe 2 : une érection insuffisante (rigidité réduite);

- groupe 3 : une érection normale (rigidité normale).

\section{Résultats}

Le priapisme drépanocytaire représente $62,5 \%$ des priapismes traités dans le service d'urologie. L'âge des patients variait de 7 à 32 ans, avec une moyenne de 15,5 ans. Le délai de prise en charge thérapeutique variait de 6 à 48 heures.

Les facteurs de survenue étaient dix fois le sommeil, cinq fois la fièvre, quatre fois les crises vaso-occlusives et une fois le rapport sexuel. Des crises de priapisme intermittent ont été retrouvées à l'anamnèse chez neuf patients avant l'épisode aigu ayant conduit à l'hospitalisation.

Le taux d'hémoglobine le plus élevé était à $8,4 \mathrm{~g} / \mathrm{dl}$, et le plus bas à $4,3 \mathrm{~g} / \mathrm{dl}$. Le phénotype hémoglobinique était SS chez tous les malades.

$\mathrm{Au}$ plan thérapeutique, en première intention, cinq patients ont été traités par l'étiléfrine orale sans succès, cinq patients traités par étiléfrine intracaverneuse avec trois succès, et enfin dix patients traités par fistule cavernospongieuse selon la technique de Winter, avec sept succès. En deuxième intention, les sept échecs par l'étiléfrine ont été traités par la technique de Winter, alors que les trois échecs de la technique de Winter ont été traités par celle d'Al-Ghorab (Tableau 1).

Au plan fonctionnel, à long terme (12 mois), nous avons obtenu cinq ( $25 \%$ ) absences d'érection, cinq ( $25 \%$ ) insuffisances d'érection et dix (50\%) érections normales.

Parmi les dix patients ayant une absence ou une insuffisance d'érection, tous avaient été traités après la $30^{\mathrm{e}}$ heure, et en deuxième intention selon les techniques de Winter ou d'Al-Ghorab.

\section{Discussion}

La prévalence de la drépanocytose, toutes formes confondues en Afrique noire, varie de 10 à $30 \%$ [7]. Le priapisme est une complication rare retrouvée chez 4 à $5 \%$ des drépanocytaires [8].

Le mécanisme physiopathologique pourrait être la survenue d'une crise de falciformation sans hémolyse. La 


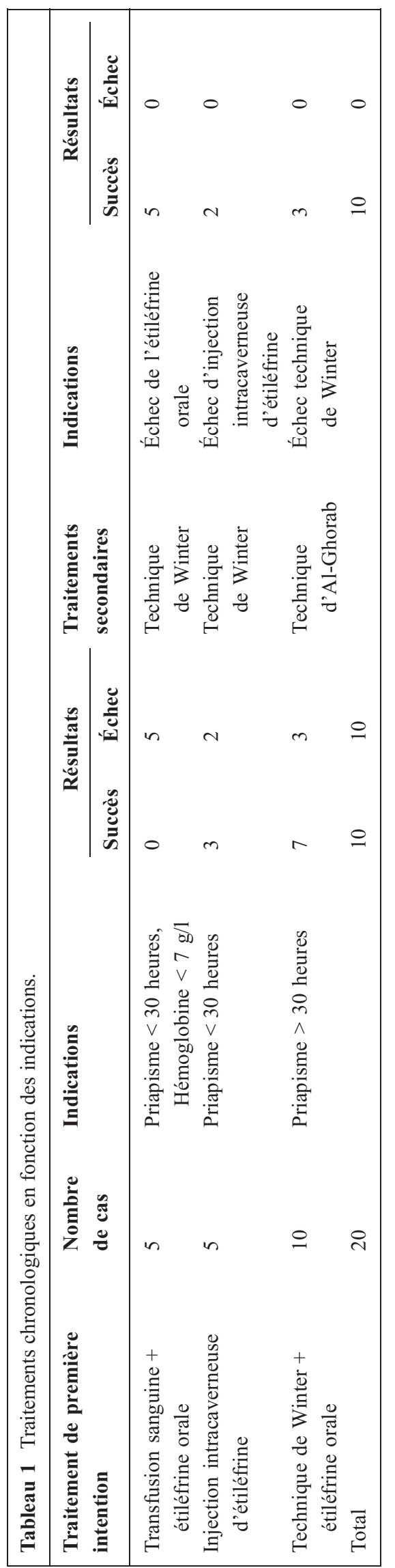

congestion veineuse ou la vagotonie du sommeil entraînerait un ralentissement circulatoire, avec baisse de la $\mathrm{PO}_{2}$, facteur de falciformation, elle-même aggravant la stase et favorisant les thromboses [9]. Il s'agit ici d'une thrombose des corps caverneux suite à leur obstruction par les drépanocytes, entraînant une stase sanguine, une hypoxie et une acidose.

Les principaux facteurs favorisants retrouvés dans notre étude sont le sommeil, la fièvre et les crises vaso-occlusives. Pendant le sommeil, il survient des érections mais aussi un ralentissement de la circulation avec baisse de la pression partielle en oxygène $[7,10]$.

Les différents traitements découlent de la connaissance de ce mécanisme physiologique et tiennent aussi compte de la durée du priapisme. D'après les travaux de Spycher et Hauri, il existerait dans les priapismes de bas débit, ce qui est le cas dans la drépanocytose, une nécrose des cellules endothéliales et du tissu érectile à partir de la $24^{\mathrm{e}}$ heure [10]. Le priapisme drépanocytaire est le plus souvent un priapisme veineux ou de stase qui nécessite un traitement urgent médical ou par ponction lavage et injection intracaverneuse de substances agonistes sélectives alpha-1 adrénergiques.

Ainsi, le traitement par injection intracaverneuse d'étiléfrine, réalisé en première intention chez cinq patients de notre série avant 30 heures, a donné deux récidives chez un patient vu à 16 heures et un autre à 20 heures. Les trois succès sont observés chez des patients vus avant la $24^{\mathrm{e}}$ heure.

Dans la série de Gbadoe et al. [11], la détumescence a été obtenue dans tous les cas chez les patients traités avant la $29^{\mathrm{e}}$ heure, et dans $22 \%$ des cas chez les patients traités après la $29^{\mathrm{e}}$ heure. L'étiléfrine par voie orale associée à la transfusion sanguine et l'hydratation ont été inefficaces dans toutes les tentatives en première intention dans notre étude.

Cette substance utilisée depuis 1994 dans le traitement du priapisme semble plus efficace par voie injectable que par voie orale [12]. Les injections intracaverneuses d'étiléfrine sont à utiliser en première intention avant 24 heures. Au-delà de 24 heures, les méthodes de drainage par shunt semblent plus efficaces et mieux indiquées [13]. La technique de Winter, par sa simplicité, le respect de l'anatomie et la faible morbidité, est la plus utilisée [14]. Celle d'Al-Ghorab est souvent utilisée en deuxième intention, après échec de la précédente. Dans notre étude, la prévention des récidives a été assurée par l'administration de l'étiléfrine orale. Dans de récentes publications, le finastéride ainsi que l'association kétoconazole-prédnisone ont été utilisés avec des résultats satisfaisants sur la prévention des récidives et la préservation de la fonction érectile $[15,16]$.

À distance, le taux d'impuissance définitive a été de $25 \%$ et celui d'insuffisance érectile à $25 \%$ dans notre série. Ce taux d'impuissance secondaire est de $56,8 \%$ dans la série de Falandry après traitement chirurgical du priapisme de toute étiologie confondue en milieu africain [14]. 
Le délai de consultation est un élément important du pronostic. Pour la majorité des auteurs, il ne devrait pas dépasser 36 heures pour minimiser les risques d'impuissance érectile [17]. Ce risque atteindrait $60 \%$ après 48 heures et dépasserait $80 \%$ après quatre jours [18].

\section{Conclusion}

L'étiologie drépanocytaire est prédominante chez nos malades traités pour priapisme. Ainsi, devant un priapisme drépanocytaire, le traitement par ponction drainage et injection intracaverneuse d'étiléfrine semble efficace et justifié avant la $24^{\mathrm{e}}$ heure. Au-delà de ce délai, les dérivations par fistule cavernospongieuse sont plus indiquées et donnent les meilleurs résultats. La sensibilisation de la population sur la maladie drépanocytaire et ses complications constitue l'un des principaux moyens de prévention. La connaissance des indications thérapeutiques et la prise en charge précoce permettent d'améliorer le pronostic fonctionnel.

\section{Conflit d'intérêt : aucun.}

\section{Références}

1. Jolidon C, Cordier M, Lanson Y (1985) Le priapisme à travers les siècles. J Urol (Paris) 91(4):225-6

2. Elira Dokekias A, Nzingoula S (2001) Profil du sujet drépanocytaire homozygote après l'âge de 30 ans. Med Afr Noire 48:411-8

3. Djembo-Taty M, Tchiloemba M, Galacteros F, et al (1986) Étude épidémiologique des hémoglobinopathies au Congo chez 2257 nouveau-nés. Nouv Rev Fr Hematol 28(4):249-51
4. Arnal C, Girot R (2002) Drépanocytose chez l'adulte. Encycl Med Chir (Éditions scientifiques et Médicales Elsevier SAS, Paris), Hématologie, 13-006-D-15

5. Ramos CE, Park JS, Ritchey ML, Benson GS (1995) High flow priapism associated with sickle cell disease. J Urol 153:1619-21

6. Buchanan GR, DeBaun MR, Quinn CT, Steinberg MH (2004) Sickle cell disease. Hematology Am Soc Hematol Educ Program $35-47$

7. Roupret M, Beley S, Traxer O, et al (2005) Prise en charge du priapisme chez les patients drépanocytaires. Prog Urol 15(3):392-6

8. Sarramon JP, Lhez JM, Leandri P, Rossignol G (1983) Priapisme. Encycl Med Chir (éditions Techniques, Paris) Rein. Organes génito-urinaires, 18-390 B20 : 11p

9. Rudelle E, Bitker MO, Saad H, Chatelain C (1993) Priapisme. Encycl Med Chir (Paris France), Urologie, 18-380-A-10, 5p

10. Spycher MA, Hauri D (1986) The ultrastructure of the erectile tissue in priapism. J Urol 135(1):142-7

11. Gbadoe AD, Akakpo-Vidah A, Pitche P, Assimadi JK (2000) Priapisme drépanocytaire : prise en charge par injections intracaverneuses d'étiléfrine. Med Trop 60:53-6

12. Mantadakis E, Ewalt DH, Cavender JD, et al (2000) Outpatient penile aspiration and epinephrine irrigation for young patients with sickle cell anemia and prolonged priapism. Blood 95(1): $78-82$

13. Falandry L (2001) Traitement chirurgical du priapisme par ponction drainage balanique à « ciel ouvert » sur 26 patients de race noire. Ann Urol (Paris) 35(3):172-7

14. Falandry L, Berlizot P, Fournier R, et al (2000). Traitement chirurgical du priapisme : expérience de 56 cas en milieu africain. Med Trop 60:70-4

15. Rachid-Filho D, Cavalcanti AG, Favorito LA, et al (2009) Treatment of recurrent priapism in sickle cell anemia with finasteride: a new approach. Urology 74(5):1054-7

16. Abern MR, Levine LA (2009) Ketoconazole and prednisone to prevent recurrent ischemic priapism. J Urol 182(4):1401-6

17. Falandry L (1999) Priapisme : traitement et résultats. À propos d'une série personnelle de 56 cas. Prog Urol 9(3):496-501

18. Bouya PA, Lomina D (2005) Prise en charge du priapisme : à propos de 32 cas. Med Afr Noire 52:453-6 